\title{
Lazer-Leach type condition for second order differential equations at resonance with impulsive effects via variational method
}

$\operatorname{Jin} L i^{*}$

\section{"Correspondence:}

lijin7912@gmail.com

School of Science, Jiujiang

University, Jiujiang, 332005, China

\begin{abstract}
In this paper, we study the existence of periodic solutions of second order impulsive differential equations at resonance with impulsive effects. We prove the existence of periodic solutions under a generalized Lazer-Leach type condition by using variational method. The impulses can generate a periodic solution.
\end{abstract}

Keywords: impulsive differential equations; Lazer-Leach type condition; variational method

\section{Introduction}

We are concerned with the periodic boundary value problem of second order impulsive differential equations at resonance

$$
\left\{\begin{array}{l}
x^{\prime \prime}(t)+m^{2} x(t)+g(x(t))=e(t), \quad \text { a.e. } t \in[0,2 \pi], \\
x(0)-x(2 \pi)=x^{\prime}(0)-x^{\prime}(2 \pi)=0, \\
x\left(t_{j}^{+}\right)=x\left(t_{j}^{-}\right), \\
\Delta x^{\prime}\left(t_{j}\right):=x^{\prime}\left(t_{j}^{+}\right)-x^{\prime}\left(t_{j}^{-}\right)=I_{j}\left(t_{j}, x\left(t_{j}\right)\right), \quad j=1,2, \ldots, p,
\end{array}\right.
$$

where $m \in \mathbb{N}, g: \mathbb{R} \rightarrow \mathbb{R}$ is a continuous function, $e \in L^{1}(0,2 \pi), 0<t_{1}<t_{2}<\cdots<t_{p}<2 \pi$, and $I_{j}:[0,2 \pi] \times \mathbb{R} \rightarrow \mathbb{R}$ is continuous for every $j$.

When $\Delta x^{\prime}\left(t_{j}\right) \equiv 0$, problem (1.1) becomes the well-known periodic boundary value problem at resonance

$$
\left\{\begin{array}{l}
x^{\prime \prime}(t)+m^{2} x(t)+g(x(t))=e(t), \quad \text { a.e. } t \in[0,2 \pi], \\
x(0)-x(2 \pi)=x^{\prime}(0)-x^{\prime}(2 \pi)=0 .
\end{array}\right.
$$

Assume that

$$
\lim _{x \rightarrow \pm \infty} g(x)=g( \pm \infty)
$$

exist and are finite. Lazer and Leach [1] proved that (1.2) has at least one $2 \pi$-periodic solution provided that the following condition holds:

$$
2[g(+\infty)-g(-\infty)] \neq \int_{0}^{2 \pi} e(t) \sin (m t+\theta) d t, \quad \forall \theta \in \mathbb{R} .
$$

\section{Springer}

2014 Li: licensee Springer. This is an Open Access article distributed under the terms of the Creative Commons Attribution License (http://creativecommons.org/licenses/by/4.0), which permits unrestricted use, distribution, and reproduction in any medium, provided the original work is properly credited. 
From then on, a series of relevant resonant problems were studied (see [2-5] and the references cited therein) by some classical tools such as topological degree method, variational method, etc. Recently, the periodic problem of the second order differential equation with impulses has been widely studied because of its background in applied sciences (see [6-18] and the references cited therein). In this paper, we investigate problem (1.1) under a more general Lazer-Leach type condition. Define

$$
G(x)=\int_{0}^{x} g(s) d s
$$

and for $j=1,2, \ldots, p$,

$$
J_{j}(t, x)=\int_{0}^{x} I_{j}(t, s) d s
$$

Throughout this paper, we give the following fundamental assumptions.

$\left(\mathrm{H}_{1}\right)$ The limits

$$
\lim _{x \rightarrow \pm \infty} \frac{G(x)}{x}=G( \pm \infty)
$$

exist and are finite.

$\left(\mathrm{H}_{2}\right)$ There exist continuous, $2 \pi$-periodic functions $K_{1}(t), K_{2}(t), \ldots, K_{p}(t)$ such that for $j=$ $1,2, \ldots, p$,

$$
\lim _{|x| \rightarrow \infty} \frac{I_{j}(t, x)}{x}=K_{j}(t) \quad \text { uniformly for } t \in \mathbb{R} .
$$

$\left(\mathrm{H}_{3}\right)$ For all $\theta \in \mathbb{R}$,

$$
2[G(+\infty)-G(-\infty)] \neq \int_{0}^{2 \pi} e(t) \sin (m t+\theta) d t+\sum_{j=1}^{p} K_{j}\left(t_{j}\right) \sin \left(m t_{j}+\theta\right)
$$

For the sake of convenience, we decompose $\left(\mathrm{H}_{3}\right)$ into the following two conditions.

$\left(\mathrm{H}_{3}^{+}\right)$For all $\theta \in \mathbb{R}$,

$$
2[G(+\infty)-G(-\infty)]>\int_{0}^{2 \pi} e(t) \sin (m t+\theta) d t+\sum_{j=1}^{p} K_{j}\left(t_{j}\right) \sin \left(m t_{j}+\theta\right)
$$

$\left(\mathrm{H}_{3}^{-}\right)$For all $\theta \in \mathbb{R}$,

$$
2[G(+\infty)-G(-\infty)]<\int_{0}^{2 \pi} e(t) \sin (m t+\theta) d t+\sum_{j=1}^{p} K_{j}\left(t_{j}\right) \sin \left(m t_{j}+\theta\right)
$$

We now can state the main theorems of this paper.

Theorem 1.1 Assume that conditions $\left(\mathrm{H}_{1}\right),\left(\mathrm{H}_{2}\right)$ and $\left(\mathrm{H}_{3}^{+}\right)$hold. Then problem (1.1) has at least one $2 \pi$-periodic solution. 
Theorem 1.2 Assume that conditions $\left(\mathrm{H}_{1}\right),\left(\mathrm{H}_{2}\right)$ and $\left(\mathrm{H}_{3}^{-}\right)$hold. Then problem (1.1) has at least one $2 \pi$-periodic solution.

From Theorem 1.1 and Theorem 1.2, we obtain the following theorem.

Theorem 1.3 Assume that conditions $\left(\mathrm{H}_{1}\right),\left(\mathrm{H}_{2}\right)$ and $\left(\mathrm{H}_{3}\right)$ hold. Then problem (1.1) has at least one $2 \pi$-periodic solution.

Moreover, we have the following corollary.

Corollary 1.4 Assume that conditions $\left(\mathrm{H}_{1}\right)$ and

$\left(\mathrm{H}_{3}^{\prime}\right)$ for all $\theta \in \mathbb{R}$,

$$
2[G(+\infty)-G(-\infty)] \neq \int_{0}^{2 \pi} e(t) \sin (m t+\theta) d t
$$

hold. Then problem (1.2) has at least one $2 \pi$-periodic solution.

Remark 1.5 It is easy to find a function $g(x)$ such that $(\mathrm{g})$ is not satisfied and $\left(\mathrm{H}_{1}\right)$ holds. For example, we can take $g(x)=\cos x$. Hence, Corollary 1.4 improves the related results in the literature mentioned above. Moreover, since we consider the problem with impulses, Theorem 1.3 is also a complement of the pioneering works.

Remark 1.6 When condition $\left(\mathrm{H}_{3}^{\prime}\right)$ is not satisfied, i.e., there exists $\theta_{0} \in \mathbb{R}$ such that

$$
2[G(+\infty)-G(-\infty)]=\int_{0}^{2 \pi} e(t) \sin \left(m t+\theta_{0}\right) d t
$$

problem (1.2) may have no solution. For example, we consider the resonant differential equation

$$
x^{\prime \prime}+m^{2} x+\arctan x=4 \cos m t .
$$

Obviously, $g(x)=\arctan x, e(t)=4 \cos m t$ and $G(+\infty)=\frac{\pi}{2}, G(-\infty)=-\frac{\pi}{2}$. We have

$$
\begin{aligned}
& 2[G(+\infty)-G(-\infty)]-\int_{0}^{2 \pi} e(t) \sin (m t+\theta) d t \\
& \quad=2 \pi-4 \int_{0}^{2 \pi} \cos m t \sin (m t+\theta) d t \\
& \quad=2 \pi-4 \pi \sin \theta .
\end{aligned}
$$

We take $\theta_{0} \in \mathbb{R}$ such that $\sin \theta_{0}=\frac{1}{2}$. Then $\left(\mathrm{H}_{3}^{\prime}\right)$ is not satisfied. From now on, we prove that (1.5) has no $2 \pi$-periodic solution by contradiction. Assume that (1.5) has $2 \pi$-periodic solution. Multiplying both sides of (1.5) by $\cos m t$ and integrating over $[0,2 \pi]$, we get

$$
4 \pi=\int_{0}^{2 \pi} \arctan x \cos m t d t \leq \int_{0}^{2 \pi}|\arctan x \cos m t| d t \leq \frac{\pi}{2} \int_{0}^{2 \pi} d t=\pi^{2},
$$


which is impossible. Hence, problem (1.2) may have no solution if condition $\left(\mathrm{H}_{3}^{\prime}\right)$ is not satisfied. Now, we give the following boundary value condition:

$$
x(0)-x(2 \pi)=x^{\prime}(0)-x^{\prime}(2 \pi)=0
$$

and the impulsive condition

$$
\Delta x^{\prime}\left(\frac{\pi}{m}\right)=3 \pi \text {. }
$$

Clearly, $p=1$ and $K_{1}\left(\frac{\pi}{m}\right)=3 \pi$. Then

$$
\begin{aligned}
& 2[G(+\infty)-G(-\infty)]-\int_{0}^{2 \pi} e(t) \sin (m t+\theta) d t-K_{1}\left(\frac{\pi}{m}\right) \sin \left(m \cdot \frac{\pi}{m}+\theta\right) \\
& \quad=2 \pi-4 \pi \sin \theta+3 \pi \sin \theta \\
& \quad=2 \pi-\pi \sin \theta \neq 0 \quad \text { for } \forall \theta \in \mathbb{R} .
\end{aligned}
$$

Hence, $\left(\mathrm{H}_{1}\right),\left(\mathrm{H}_{2}\right)$ and $\left(\mathrm{H}_{3}\right)$ hold. Equivalently, Eq. (1.5) with conditions (1.6) and (1.7) has at least one $2 \pi$-periodic solution. Therefore, the impulses in problem (1.1) can generate a periodic solution.

The rest of the paper is organized as follows. In Section 2, we shall state some notations, some necessary definitions and a saddle theorem due to Rabinowitz. In Section 3, we shall prove Theorem 1.1 and Theorem 1.2.

\section{Preliminaries}

In the following, we introduce some notations and some necessary definitions.

Define

$$
H=\left\{x \in H^{1}(0,2 \pi): x(0)=x(2 \pi)\right\},
$$

with the norm

$$
\|x\|=\left(\int_{0}^{2 \pi}\left(x^{\prime 2}(t)+x^{2}(t)\right) d t\right)^{\frac{1}{2}} .
$$

Consider the functional $\varphi(x)$ defined on $H$ by

$$
\begin{aligned}
\varphi(x)= & \frac{1}{2} \int_{0}^{2 \pi} x^{\prime 2}(t) d t-\frac{m^{2}}{2} \int_{0}^{2 \pi} x^{2}(t) d t-\int_{0}^{2 \pi} G(x(t)) d t \\
& +\int_{0}^{2 \pi} e(t) x(t) d t+\sum_{j=1}^{p} J_{j}\left(t_{j}, x\left(t_{j}\right)\right) .
\end{aligned}
$$

Similarly as in [18], $\varphi(x)$ is continuously differentiable on $H$, and

$$
\begin{aligned}
\varphi^{\prime}(x) \nu(t)= & \int_{0}^{2 \pi} x^{\prime}(t) v^{\prime}(t) d t-m^{2} \int_{0}^{2 \pi} x(t) v(t) d t-\int_{0}^{2 \pi} g(x(t)) v(t) d t \\
& +\int_{0}^{2 \pi} e(t) v(t) d t+\sum_{j=1}^{p} I_{j}\left(t_{j}, x\left(t_{j}\right)\right) v\left(t_{j}\right) \quad \text { for } \forall v(t) \in H .
\end{aligned}
$$


Now, we have the following lemma.

Lemma 2.1 If $x \in H$ is a critical point of $\varphi$, then $x$ is a $2 \pi$-periodic solution of Eq. (1.1).

The proof of Lemma 2.1 is similar to Lemma 2.1 in [9], so we omit it.

We say that $\varphi$ satisfies (PS) if every sequence $\left(x_{n}\right)$ for which $\varphi\left(x_{n}\right)$ is bounded in $\mathbb{R}$ and $\varphi^{\prime}\left(x_{n}\right) \rightarrow 0($ as $n \rightarrow \infty)$ possesses a convergent subsequence.

To prove the main result, we will use the following saddle point theorem due to Rabinowitz [19] (or see [20]).

Theorem 2.2 Let $\varphi \in C^{1}(H, \mathbb{R})$ and $H=H^{-} \oplus H^{+}, \operatorname{dim}\left(H^{-}\right)<\infty, \operatorname{dim}\left(H^{+}\right)=\infty$. We suppose that:

(a) there exist a bounded neighborhood $D$ of 0 in $H^{-}$and a constant $\alpha$ such that $\left.\varphi\right|_{\partial D} \leq \alpha$

(b) there exists a constant $\beta>\alpha$ such that $\left.\varphi\right|_{H^{+}} \geq \beta$;

(c) $\varphi$ satisfies (PS).

Then the functional $\varphi$ has a critical point in $H$.

\section{The proof of the main results}

In this section, we first show that the functional $\varphi$ satisfies the Palais-Smale condition.

Lemma 3.1 Assume that conditions $\left(\mathrm{H}_{1}\right),\left(\mathrm{H}_{2}\right)$ and $\left(\mathrm{H}_{3}\right)$ hold. Then $\varphi$ defined by $(2.1)$ satisfies (PS).

Proof Let $M>0$ be a constant and $\left\{x_{n}\right\} \subset H$ be a sequence satisfying

$$
\begin{aligned}
\left|\varphi\left(x_{n}\right)\right|= & \mid \frac{1}{2} \int_{0}^{2 \pi} x_{n}^{\prime 2} d t-\frac{m^{2}}{2} \int_{0}^{2 \pi} x_{n}^{2} d t-\int_{0}^{2 \pi} G\left(x_{n}\right) d t \\
& +\int_{0}^{2 \pi} e(t) x_{n}(t) d t+\sum_{j=1}^{p} J_{j}\left(t_{j}, x_{n}\left(t_{j}\right)\right) \mid \\
\leq & M
\end{aligned}
$$

and

$$
\lim _{n \rightarrow \infty}\left\|\varphi^{\prime}\left(x_{n}\right)\right\|=0
$$

We first prove that $\left\{x_{n}\right\}$ is bounded in $H$ by contradiction. Assume that $\left\{x_{n}\right\}$ is unbounded. Let $\left\{z_{k}\right\}$ be an arbitrary sequence bounded in $H$. It follows from (3.2) that, for any $k \in \mathbb{N}$,

$$
\lim _{n \rightarrow \infty}\left|\varphi^{\prime}\left(x_{n}\right) z_{k}\right| \leq \lim _{n \rightarrow \infty}\left\|\varphi^{\prime}\left(x_{n}\right)\right\|\left\|z_{k}\right\|=0 .
$$

Thus

$$
\lim _{n \rightarrow \infty} \varphi^{\prime}\left(x_{n}\right) z_{k}=0 \quad \text { uniformly for } k \in \mathbb{N} \text {. }
$$


Hence

$$
\begin{aligned}
& \lim _{n \rightarrow \infty}\left(\int_{0}^{2 \pi}\left(x_{n}^{\prime} z_{k}^{\prime}-m^{2} x_{n} z_{k}\right) d t-\int_{0}^{2 \pi}\left(g\left(x_{n}\right) z_{k}-e(t) z_{k}\right) d t\right. \\
& \left.+\sum_{j=1}^{p} I_{j}\left(t_{j}, x_{n}\left(t_{j}\right)\right) z_{k}\left(t_{j}\right)\right)=0 .
\end{aligned}
$$

By $\left(\mathrm{H}_{1}\right)$ and $\left(\mathrm{H}_{2}\right)$, we have

$$
\lim _{n \rightarrow \infty}\left(\int_{0}^{2 \pi} \frac{g\left(x_{n}\right) z_{k}-e(t) z_{k}}{\left\|x_{n}\right\|} d t-\frac{\sum_{j=1}^{p} I_{j}\left(t_{j}, x_{n}\left(t_{j}\right)\right) z_{k}\left(t_{j}\right)}{\left\|x_{n}\right\|}\right)=0 .
$$

From (3.3) and (3.4), we obtain

$$
\lim _{n \rightarrow \infty} \int_{0}^{2 \pi}\left(\frac{x_{n}^{\prime}}{\left\|x_{n}\right\|} z_{k}^{\prime}-m^{2} \frac{x_{n}}{\left\|x_{n}\right\|} z_{k}\right) d t=0
$$

Set

$$
y_{n}=\frac{x_{n}}{\left\|x_{n}\right\|} .
$$

Then we have

$$
\lim _{n \rightarrow \infty} \int_{0}^{2 \pi}\left(y_{n}^{\prime} z_{k}^{\prime}-m^{2} y_{n} z_{k}\right) d t=0
$$

and furthermore,

$$
\lim _{\substack{n \rightarrow \infty \\ i \rightarrow \infty}} \int_{0}^{2 \pi}\left[\left(y_{n}-y_{i}\right)^{\prime} z_{k}^{\prime}-m^{2}\left(y_{n}-y_{i}\right) z_{k}\right] d t=0
$$

Replacing $z_{k}$ in (3.6) by $\left(y_{n}-y_{i}\right)$, we get

$$
\lim _{\substack{n \rightarrow \infty \\ i \rightarrow \infty}}\left(\left\|y_{n}-y_{i}\right\|^{2}-\left(m^{2}+1\right)\left\|y_{n}-y_{i}\right\|_{2}^{2}\right)=0 .
$$

Due to the compact imbedding $H \hookrightarrow L^{2}(0,2 \pi)$, going to a subsequence,

$$
y_{n} \rightarrow y_{0} \quad \text { weakly in } H, \quad y_{n} \rightarrow y_{0} \quad \text { in } L^{2}(0,2 \pi) .
$$

Therefore,

$$
\lim _{\substack{n \rightarrow \infty \\ i \rightarrow \infty}}\left\|y_{n}-y_{i}\right\|_{2}^{2}=0
$$

Furthermore, we have

$$
\lim _{\substack{n \rightarrow \infty \\ i \rightarrow \infty}}\left\|y_{n}-y_{i}\right\|^{2}=0
$$


which implies that $\left\{y_{n}\right\}$ is a Cauchy sequence in $H$. Thus, $y_{n} \rightarrow y_{0}$ in $H$. It follows from (3.5) and the usual regularity argument for ordinary differential equations (see [21]) that

$$
y_{0}=k_{1} \sin m t+k_{2} \cos m t,
$$

where $k_{1}^{2}+k_{2}^{2}=\frac{1}{\left(m^{2}+1\right) \pi}\left(\left\|y_{0}\right\|=1\right)$. (Different subsequences of $\left\{y_{n}\right\}$ correspond to different $k_{1}$ and $k_{2}$.)

Write (3.7) as

$$
y_{0}=\frac{1}{\sqrt{\left(m^{2}+1\right) \pi}} \sin (m t+\theta)
$$

where $\theta$ satisfies $\sin \theta=\frac{k_{2}}{\sqrt{k_{1}^{2}+k_{2}^{2}}}$ and $\cos \theta=\frac{k_{1}}{\sqrt{k_{1}^{2}+k_{2}^{2}}}$.

Taking $z_{k}=\frac{1}{\sqrt{\left(m^{2}+1\right) \pi}} \sin (m t+\theta)$, we get, for any $n \in \mathbb{N}$,

$$
\int_{0}^{2 \pi}\left(x_{n}^{\prime} z_{k}^{\prime}-m^{2} x_{n} z_{k}\right) d t=0
$$

Thus, it follows from (3.3) and (3.8) that

$$
\begin{gathered}
\lim _{n \rightarrow \infty}\left[\int_{0}^{2 \pi}\left(g\left(x_{n}\right)-e(t)\right) \frac{1}{\sqrt{\left(m^{2}+1\right) \pi}} \sin (m t+\theta) d t\right. \\
\left.-\sum_{j=1}^{p} I_{j}\left(t_{j}, x_{n}\left(t_{j}\right)\right) \frac{1}{\sqrt{\left(m^{2}+1\right) \pi}} \sin \left(m t_{j}+\theta\right)\right]=0 .
\end{gathered}
$$

By $\left(\mathrm{H}_{1}\right)$ and $\left(\mathrm{H}_{2}\right)$, we obtain

$$
\begin{aligned}
\lim _{n \rightarrow \infty} & {\left[\int_{0}^{2 \pi}\left(g\left(x_{n}\right)-e(t)\right)\left(\frac{1}{\sqrt{\left(m^{2}+1\right) \pi}} \sin (m t+\theta)-y_{n}\right) d t\right.} \\
& \left.-\sum_{j=1}^{p} I_{j}\left(t_{j}, x_{n}\left(t_{j}\right)\right)\left(\frac{1}{\sqrt{\left(m^{2}+1\right) \pi}} \sin \left(m t_{j}+\theta\right)-y_{n}\left(t_{j}\right)\right)\right]=0 .
\end{aligned}
$$

It follows from (3.9) and (3.10) that

$$
\lim _{n \rightarrow \infty}\left[\int_{0}^{2 \pi}\left(g\left(x_{n}\right)-e(t)\right) y_{n} d t-\sum_{j=1}^{p} I_{j}\left(t_{j}, x_{n}\left(t_{j}\right)\right) y_{n}\left(t_{j}\right)\right]=0 .
$$

Hence, replacing $z_{k}$ in (3.3) by $y_{n}$, we have

$$
\lim _{n \rightarrow \infty} \int_{0}^{2 \pi}\left(x_{n}^{\prime} \frac{x_{n}^{\prime}}{\left\|x_{n}\right\|}-m^{2} x_{n} \frac{x_{n}}{\left\|x_{n}\right\|}\right) d t=0 .
$$

Now, dividing (3.1) by $\left\|x_{n}\right\|$, we get

$$
\left|\frac{1}{2} \int_{0}^{2 \pi}\left(\frac{x_{n}^{\prime 2}}{\left\|x_{n}\right\|}-\frac{m^{2} x_{n}^{2}}{\left\|x_{n}\right\|}\right) d t-\int_{0}^{2 \pi} \frac{G\left(x_{n}\right)-e(t) x_{n}}{\left\|x_{n}\right\|} d t+\frac{\sum_{j=1}^{p} J_{j}\left(t_{j}, x_{n}\left(t_{j}\right)\right)}{\left\|x_{n}\right\|}\right| \leq \frac{M}{\left\|x_{n}\right\|} .
$$


Passing to the limits, we have

$$
\begin{aligned}
0= & \lim _{n \rightarrow \infty} \frac{\int_{0}^{2 \pi}\left(G\left(x_{n}\right)-e(t) x_{n}\right) d t}{\left\|x_{n}\right\|}-\lim _{n \rightarrow \infty} \frac{\sum_{j=1}^{p} J_{j}\left(t_{j}, x_{n}\left(t_{j}\right)\right)}{\left\|x_{n}\right\|} \\
= & \lim _{n \rightarrow \infty} \int_{0}^{2 \pi} \frac{G\left(x_{n}\right)}{x_{n}} \cdot \frac{x_{n}}{\left\|x_{n}\right\|} d t-\lim _{n \rightarrow \infty} \int_{0}^{2 \pi} e(t) \cdot \frac{x_{n}}{\left\|x_{n}\right\|} d t \\
& -\lim _{n \rightarrow \infty} \sum_{j=1}^{p} \frac{J_{j}\left(t_{j}, x_{n}\left(t_{j}\right)\right)}{x_{n}\left(t_{j}\right)} \cdot \frac{x_{n}\left(t_{j}\right)}{\left\|x_{n}\right\|} .
\end{aligned}
$$

Noting that $\frac{x_{n}}{\left\|x_{n}\right\|} \rightarrow \frac{1}{\sqrt{\left(m^{2}+1\right) \pi}} \sin (m t+\theta)$ in $H$ as $n \rightarrow \infty$ and

$$
\lim _{n \rightarrow \infty} x_{n}(t)= \begin{cases}+\infty, & \forall t \in I_{+} \\ -\infty, & \forall t \in I_{-}\end{cases}
$$

where $I_{+}:=\{t \in[0,2 \pi] \mid \sin (m t+\theta)>0\}, I_{-}:=\{t \in[0,2 \pi] \mid \sin (m t+\theta)<0\}$, we get from the Lebesgue domain convergence theorem that

$$
\begin{aligned}
0= & \int_{I_{+}} G(+\infty) \frac{1}{\sqrt{\left(m^{2}+1\right) \pi}} \sin ^{+}(m t+\theta) d t-\int_{I_{+}} G(-\infty) \frac{1}{\sqrt{\left(m^{2}+1\right) \pi}} \sin ^{-}(m t+\theta) d t \\
& -\int_{0}^{2 \pi} e(t) \frac{1}{\sqrt{\left(m^{2}+1\right) \pi}} \sin (m t+\theta) d t-\sum_{j=1}^{p} K_{j}\left(t_{j}\right) \frac{1}{\sqrt{\left(m^{2}+1\right) \pi}} \sin \left(m t_{j}+\theta\right),
\end{aligned}
$$

i.e.,

$$
0=2[G(+\infty)-G(-\infty)]-\int_{0}^{2 \pi} e(t) \sin (m t+\theta) d t-\sum_{j=1}^{p} K_{j}\left(t_{j}\right) \sin \left(m t_{j}+\theta\right),
$$

which contradicts $\left(\mathrm{H}_{3}\right)$. This implies that the sequence $\left\{x_{n}\right\}$ is bounded. Thus, there exists $x_{0} \in H$ such that $x_{n} \rightarrow x_{0}$ weakly in $H$. Due to the compact imbedding $H \hookrightarrow L^{2}(0,2 \pi)$ and $H \hookrightarrow C(0,2 \pi)$, going to a subsequence,

$$
x_{n} \rightarrow x_{0} \quad \text { in } L^{2}(0,2 \pi), \quad x_{n} \rightarrow x_{0} \quad \text { in } C(0,2 \pi) .
$$

From (3.3), we obtain

$$
\begin{aligned}
& \lim _{\substack{n \rightarrow \infty \\
i \rightarrow \infty}}\left(\int_{0}^{2 \pi}\left(\left(x_{n}^{\prime}-x_{i}^{\prime}\right) z_{k}^{\prime}-m^{2}\left(x_{n}-x_{i}\right) z_{k}\right) d t-\int_{0}^{2 \pi}\left(g\left(x_{n}\right)-g\left(x_{i}\right)\right) z_{k} d t\right. \\
& \left.\quad+\sum_{j=1}^{p}\left(I_{j}\left(t_{j}, x_{n}\left(t_{j}\right)\right)-I_{j}\left(t_{j}, x_{i}\left(t_{j}\right)\right)\right) z_{k}\left(t_{j}\right)\right)=0 .
\end{aligned}
$$

Replacing $z_{k}$ by $x_{n}-x_{i}$ in the above equality, we get

$$
\begin{aligned}
& \lim _{\substack{n \rightarrow \infty \\
i \rightarrow \infty}}\left(\int_{0}^{2 \pi}\left(\left(x_{n}^{\prime}-x_{i}^{\prime}\right)^{2}-m^{2}\left(x_{n}-x_{i}\right)^{2}\right) d t-\int_{0}^{2 \pi}\left(g\left(x_{n}\right)-g\left(x_{i}\right)\right)\left(x_{n}-x_{i}\right) d t\right. \\
& \left.\quad+\sum_{j=1}^{p}\left(I_{j}\left(t_{j}, x_{n}\left(t_{j}\right)\right)-I_{j}\left(t_{j}, x_{i}\left(t_{j}\right)\right)\right)\left(x_{n}\left(t_{j}\right)-x_{i}\left(t_{j}\right)\right)\right)=0 .
\end{aligned}
$$


By $\left(\mathrm{H}_{1}\right)$ and $\left(\mathrm{H}_{2}\right)$, we have

$$
\lim _{\substack{n \rightarrow \infty \\ i \rightarrow \infty}} \int_{0}^{2 \pi}\left(g\left(x_{n}\right)-g\left(x_{i}\right)\right)\left(x_{n}-x_{i}\right) d t=0
$$

and

$$
\lim _{\substack{n \rightarrow \infty \\ i \rightarrow \infty}} \sum_{j=1}^{p}\left(I_{j}\left(t_{j}, x_{n}\left(t_{j}\right)\right)-I_{j}\left(t_{j}, x_{i}\left(t_{j}\right)\right)\right)\left(x_{n}\left(t_{j}\right)-x_{i}\left(t_{j}\right)\right)=0 .
$$

Thus, it follows from (3.12), (3.13) and (3.14) that

$$
\lim _{\substack{n \rightarrow \infty \\ i \rightarrow \infty}} \int_{0}^{2 \pi}\left[\left(x_{n}^{\prime}-x_{i}^{\prime}\right)^{2}-m^{2}\left(x_{n}-x_{i}\right)^{2}\right] d t=0 .
$$

Therefore,

$$
\lim _{\substack{n \rightarrow \infty \\ i \rightarrow \infty}}\left\|x_{n}-x_{i}\right\|^{2}=0
$$

which implies $x_{n} \rightarrow x_{0}$ in $H$. It shows that $\varphi$ satisfies (PS).

Remark 3.2 If conditions $\left(\mathrm{H}_{1}\right),\left(\mathrm{H}_{2}\right)$ and $\left(\mathrm{H}_{3}^{+}\right)\left(\right.$or $\left.\left(\mathrm{H}_{3}^{-}\right)\right)$, $\varphi$ defined by (2.1) still satisfies (PS).

Now, we can give the proof of Theorem 1.1.

Proof of Theorem 1.1 Denote

$$
H^{-}=\mathbb{R} \oplus \operatorname{span}\{\sin t, \cos t, \sin 2 t, \cos 2 t, \ldots, \sin m t, \cos m t\}
$$

and

$$
H^{+}=\operatorname{span}\{\sin (m+1) t, \cos (m+1) t, \ldots\} .
$$

We first prove that

$$
\liminf _{\|x\| \rightarrow \infty} \varphi(x)=-\infty \quad \text { for } x \in H^{-}
$$

by contradiction. Assume that there exists a sequence $\left(x_{n}\right) \subset H^{-}$such that $\left\|x_{n}\right\| \rightarrow \infty$ (as $n \rightarrow \infty)$ and there exists a constant $c_{-}$satisfying

$$
\liminf _{n \rightarrow \infty} \varphi\left(x_{n}\right) \geq c_{-} .
$$

By $\left(\mathrm{H}_{1}\right)$, we have

$$
\lim _{n \rightarrow \infty} \int_{0}^{2 \pi} \frac{G\left(x_{n}\right)-e(t) x_{n}}{\left\|x_{n}\right\|^{2}} d t=0 .
$$


By $\left(\mathrm{H}_{2}\right)$, we get

$$
\lim _{n \rightarrow \infty} \sum_{j=1}^{p} \frac{J_{j}\left(t_{j}, x_{n}\left(t_{j}\right)\right)}{\left\|x_{n}\right\|^{2}}=0
$$

From (3.16) and the definition of $\varphi$, we obtain

$$
\liminf _{n \rightarrow \infty}\left[\frac{1}{2} \int_{0}^{2 \pi} \frac{x_{n}^{\prime 2}-m^{2} x_{n}^{2}}{\left\|x_{n}\right\|^{2}} d t-\int_{0}^{2 \pi} \frac{G\left(x_{n}\right)-e(t) x_{n}}{\left\|x_{n}\right\|^{2}} d t+\sum_{j=1}^{p} \frac{J_{j}\left(t_{j}, x_{n}\left(t_{j}\right)\right)}{\left\|x_{n}\right\|^{2}}\right] \geq 0
$$

For $x \in H^{-}$, we get that there exist constants $a_{0}, a_{1}, \ldots, a_{m}, b_{1}, b_{2}, \ldots, b_{m}$ such that

$$
x(t)=\sum_{j=0}^{m} a_{j} \cos j t+\sum_{j=1}^{m} b_{j} \sin j t .
$$

Since $\int_{0}^{2 \pi} \sin ^{2} j t d t=\int_{0}^{2 \pi} \cos ^{2} j t d t$ for $j=1,2, \ldots$, we have, for $x \in H^{-}$,

$$
\begin{aligned}
\int_{0}^{2 \pi} x^{\prime 2} d t & =\int_{0}^{2 \pi} \sum_{j=1}^{m} j^{2} a_{j}^{2} \sin ^{2} j t d t+\int_{0}^{2 \pi} \sum_{j=1}^{m} j^{2} b_{j}^{2} \cos ^{2} j t d t \\
& \leq m^{2}\left[\int_{0}^{2 \pi} \sum_{j=1}^{m} a_{j}^{2} \sin ^{2} j t d t+\int_{0}^{2 \pi} \sum_{j=1}^{m} b_{j}^{2} \cos ^{2} j t d t\right] \\
& =m^{2}\left[\int_{0}^{2 \pi} \sum_{j=1}^{m} a_{j}^{2} \cos ^{2} j t d t+\int_{0}^{2 \pi} \sum_{j=1}^{m} b_{j}^{2} \sin ^{2} j t d t\right] \\
& \leq m^{2} \int_{0}^{2 \pi} x^{2} d t .
\end{aligned}
$$

Hence, for $x \in H^{-}$,

$$
\int_{0}^{2 \pi}\left(x^{\prime 2}-m^{2} x^{2}\right) d t \leq 0
$$

The equality in (3.20) holds only for

$$
x=\frac{1}{\sqrt{\left(m^{2}+1\right) \pi}} \sin (m t+\theta), \quad \theta \in \mathbb{R} .
$$

Set $y_{n}=\frac{x_{n}}{\left\|x_{n}\right\|}$. Since $\operatorname{dim} H^{-}<\infty$, going to a subsequence, there exists $y_{0} \in H^{-}$such that $y_{n} \rightarrow y_{0}$ in $H$ and $y_{n} \rightarrow y_{0}$ in $L^{2}(0,2 \pi)$. Then (3.17), (3.18), (3.19) and (3.20) imply that

$$
y_{0}=\frac{1}{\sqrt{\left(m^{2}+1\right) \pi}} \sin (m t+\theta), \quad \theta \in \mathbb{R}
$$

By (3.16), we have, for $n$ large enough,

$$
\frac{1}{2} \int_{0}^{2 \pi} \frac{x_{n}^{\prime 2}-m^{2} x_{n}^{2}}{\left\|x_{n}\right\|} d t-\int_{0}^{2 \pi} \frac{G\left(x_{n}\right)-e(t) x_{n}}{\left\|x_{n}\right\|} d t+\sum_{j=1}^{p} \frac{J_{j}\left(t_{j}, x_{n}\left(t_{j}\right)\right)}{\left\|x_{n}\right\|} \geq \frac{c_{-}}{\left\|x_{n}\right\|}
$$


It follows from $x_{n} \in H^{-}$that

$$
\int_{0}^{2 \pi} \frac{x_{n}^{\prime 2}-m^{2} x_{n}^{2}}{\left\|x_{n}\right\|} \leq 0
$$

From (3.21) and (3.22), we get, for $n$ large enough,

$$
\frac{c_{-}}{\left\|x_{n}\right\|} \leq-\int_{0}^{2 \pi} \frac{G\left(x_{n}\right)-e(t) x_{n}}{\left\|x_{n}\right\|} d t+\sum_{j=1}^{p} \frac{J_{j}\left(t_{j}, x_{n}\left(t_{j}\right)\right)}{\left\|x_{n}\right\|}
$$

Passing to the limits and using an argument similarly as in the proof of Lemma 3.1, we get

$$
2[G(+\infty)-G(-\infty)] \leq \int_{0}^{2 \pi} e(t) \sin (m t+\theta) d t+\sum_{j=1}^{p} K_{j}\left(t_{j}\right) \sin \left(m t_{j}+\theta\right)
$$

which is a contradiction to $\left(\mathrm{H}_{3}^{+}\right)$.

Then (3.15) holds.

Next, we prove that

$$
\lim _{\|x\| \rightarrow \infty} \varphi(x)=\infty \quad \text { for all } x \in H^{+},
$$

and $\varphi$ is bounded on bounded sets.

Because of the compact imbedding of $H \hookrightarrow C(0,2 \pi)$ and $H \hookrightarrow L^{2}(0,2 \pi)$, there exist constants $m_{1}, m_{2}$ such that

$$
\|x\|_{\infty} \leq m_{1}\|x\|, \quad\|x\|_{2} \leq m_{2}\|x\| .
$$

Then by $\left(\mathrm{H}_{1}\right)$ and $\left(\mathrm{H}_{2}\right)$, one has that there exist positive constants $c_{g}, c_{1}, c_{2}, \ldots, c_{p}$ such that

$$
\begin{aligned}
|\varphi(x)|= & \mid \frac{1}{2} \int_{0}^{2 \pi} x^{\prime 2} d t-\frac{m^{2}}{2} \int_{0}^{2 \pi} x^{2} d t-\int_{0}^{2 \pi}[G(x)-e(t) x] d t \\
& +\sum_{j=1}^{p} J_{j}\left(t_{j}, x\left(t_{j}\right)\right) \mid \\
\leq & \frac{1}{2}\|x\|^{2}+\frac{m^{2}}{2} m_{2}^{2}\|x\|^{2}+\int_{0}^{2 \pi}\left(c_{g}|x|+|e(t)||x|\right) d t \\
& +\sum_{j=1}^{p} c_{j}\left|x\left(t_{j}\right)\right| \\
\leq & \frac{1+m^{2} m_{2}^{2}}{2}\|x\|^{2}+m_{1}\left(c_{g}+\|e\|_{1}\right)\|x\|+\sum_{j=1}^{p} c_{j} m_{1}\|x\| .
\end{aligned}
$$

Hence, $\varphi$ is bounded on the bounded sets of $H$.

For $x \in H^{+}$, using an argument similar to the case $x \in H^{-}$, we have

$$
\|x\|^{2} \geq\left((m+1)^{2}+1\right)\|x\|_{2}^{2} .
$$


Thus, from (3.23) and (3.24), we obtain

$$
\begin{aligned}
\varphi(x) & =\frac{1}{2} \int_{0}^{2 \pi} x^{\prime 2} d t-\frac{m^{2}}{2} \int_{0}^{2 \pi} x^{2} d t-\int_{0}^{2 \pi}[G(x)-e(t) x] d t+\sum_{j=1}^{p} J_{j}\left(t_{j}, x\left(t_{j}\right)\right) \\
& \geq \frac{2 m+1}{2\left((m+1)^{2}+1\right)}\|x\|^{2}-m_{1}\left(c_{g}+\|e\|_{1}+\sum_{j=1}^{p} c_{j}\right)\|x\|,
\end{aligned}
$$

which implies

$$
\lim _{\|x\| \rightarrow \infty} \varphi(x)=\infty \quad \text { for all } x \in H^{+} .
$$

Up to now, the conditions (a) and (b) of Theorem 2.2 are satisfied. According to Remark 3.2, (c) is also satisfied. Hence, by Theorem 2.2, problem (1.1) has at least one solution. This completes the proof.

Next, we prove Theorem 1.2 slightly differently from Theorem 1.1.

Proof of Theorem 1.2 Denote

$$
H^{-}=\mathbb{R} \oplus \operatorname{span}\{\sin t, \cos t, \sin 2 t, \cos 2 t, \ldots, \sin (m-1) t, \cos (m-1) t\}
$$

and

$$
H^{+}=\operatorname{span}\{\sin m t, \cos m t, \ldots\} .
$$

We first prove that

$$
\liminf _{\|x\| \rightarrow \infty} \varphi(x)=-\infty \quad \text { for } x \in H^{-}
$$

For $x \in H^{-}$, we get that there exist constants $a_{0}, a_{1}, \ldots, a_{m-1}, b_{1}, b_{2}, \ldots, b_{m-1}$ such that

$$
x(t)=\sum_{j=0}^{m-1} a_{j} \cos j t+\sum_{j=1}^{m-1} b_{j} \sin j t .
$$

Since $\int_{0}^{2 \pi} \sin ^{2} j t d t=\int_{0}^{2 \pi} \cos ^{2} j t d t$ for $j=1,2, \ldots$, we have, for $x \in H^{-}$,

$$
\begin{aligned}
\int_{0}^{2 \pi} x^{\prime 2} d t & =\int_{0}^{2 \pi} \sum_{j=1}^{m-1} j^{2} a_{j}^{2} \sin ^{2} j t d t+\int_{0}^{2 \pi} \sum_{j=1}^{m-1} j^{2} b_{j}^{2} \cos ^{2} j t d t \\
& \leq(m-1)^{2}\left[\int_{0}^{2 \pi} \sum_{j=1}^{m-1} a_{j}^{2} \sin ^{2} j t d t+\int_{0}^{2 \pi} \sum_{j=1}^{m-1} b_{j}^{2} \cos ^{2} j t d t\right] \\
& =(m-1)^{2}\left[\int_{0}^{2 \pi} \sum_{j=1}^{m-1} a_{j}^{2} \cos ^{2} j t d t+\int_{0}^{2 \pi} \sum_{j=1}^{m-1} b_{j}^{2} \sin ^{2} j t d t\right] \\
& \leq(m-1)^{2} \int_{0}^{2 \pi} x^{2} d t .
\end{aligned}
$$


Hence, for $x \in H^{-}$,

$$
\|x\|^{2}=\int_{0}^{2 \pi}\left(x^{\prime 2}+x^{2}\right) d t \leq\left[(m-1)^{2}+1\right] \int_{0}^{2 \pi} x^{2} d t=\left[(m-1)^{2}+1\right]\|x\|_{2}^{2} .
$$

The equality holds only for

$$
x=\frac{1}{\sqrt{\left((m-1)^{2}+1\right) \pi}} \sin ((m-1) t+\theta), \quad \theta \in \mathbb{R} .
$$

If $x \in H^{-}$and $\|x\| \rightarrow \infty$, then

$$
\|x\|_{2} \rightarrow \infty
$$

For $x \in H^{-}$, we have

$$
\int_{0}^{2 \pi}\left(x^{\prime 2}-m^{2} x^{2}\right) d t \leq(m-1)^{2} \int_{0}^{2 \pi} x^{2} d t-m^{2} \int_{0}^{2 \pi} x^{2} d t=-(2 m-1)\|x\|_{2}^{2} .
$$

By $\left(\mathrm{H}_{1}\right)$ and $\left(\mathrm{H}_{2}\right)$, we get that there exists a constant $c_{0}>0$ such that

$$
\left|-\int_{0}^{2 \pi}[G(x)-e(t) x] d t+\sum_{j=1}^{p} J_{j}\left(t_{j}, x\left(t_{j}\right)\right)\right| \leq c_{0}\|x\|_{2}
$$

Hence, for $x \in H^{-}$, we obtain

$$
\begin{aligned}
\varphi(x) & =\frac{1}{2} \int_{0}^{2 \pi}\left(x^{\prime 2}-m^{2} x^{2}\right) d t-\int_{0}^{2 \pi}[G(x)-e(t) x] d t+\sum_{j=1}^{p} J_{j}\left(t_{j}, x\left(t_{j}\right)\right) \\
& \leq-\frac{1}{2}(2 m-1)\|x\|_{2}^{2}+c_{0}\|x\|_{2} \rightarrow-\infty \quad \text { as }\|x\| \rightarrow \infty
\end{aligned}
$$

Therefore, (3.25) holds.

Next, we prove that

$$
\lim _{\|x\| \rightarrow \infty} \varphi(x)=\infty \quad \text { for all } x \in H^{+},
$$

and $\varphi$ is bounded on bounded sets.

Because of the compact imbedding of $H \hookrightarrow C(0,2 \pi)$ and $H \hookrightarrow L^{2}(0,2 \pi)$, there exist constants $m_{1}, m_{2}$ such that

$$
\|x\|_{\infty} \leq m_{1}\|x\|, \quad\|x\|_{2} \leq m_{2}\|x\| .
$$

Then, by $\left(\mathrm{H}_{1}\right)$ and $\left(\mathrm{H}_{2}\right)$, one has that there exist positive constants $c_{g}, c_{1}, c_{2}, \ldots, c_{p}$ such that

$$
\begin{aligned}
|\varphi(x)|= & \mid \frac{1}{2} \int_{0}^{2 \pi} x^{\prime 2} d t-\frac{m^{2}}{2} \int_{0}^{2 \pi} x^{2} d t-\int_{0}^{2 \pi}[G(x)-e(t) x] d t \\
& +\sum_{j=1}^{p} J_{j}\left(t_{j}, x\left(t_{j}\right)\right) \mid
\end{aligned}
$$




$$
\begin{aligned}
\leq & \frac{1}{2}\|x\|^{2}+\frac{m^{2}}{2} m_{2}^{2}\|x\|^{2}+\int_{0}^{2 \pi}\left(c_{g}|x|+|e(t)||x|\right) d t \\
& +\sum_{j=1}^{p} c_{j}\left|x\left(t_{j}\right)\right| \\
\leq & \frac{1+m^{2} m_{2}^{2}}{2}\|x\|^{2}+m_{1}\left(c_{g}+\|e\|_{1}\right)\|x\|+\sum_{j=1}^{p} c_{j} m_{1}\|x\| .
\end{aligned}
$$

Hence, $\varphi$ is bounded on the bounded sets of $H$.

In what follows, we prove that

$$
\lim _{\|x\| \rightarrow \infty} \varphi(x)=+\infty \quad \text { for } x \in H^{+}
$$

by contradiction. Assume that there exists a sequence $\left(x_{n}\right) \subset H^{-}$such that $\left\|x_{n}\right\| \rightarrow \infty$ (as $n \rightarrow \infty)$, and there exists a constant $c_{+}$satisfying

$$
\limsup _{n \rightarrow \infty} \varphi\left(x_{n}\right) \leq c_{+} .
$$

By $\left(\mathrm{H}_{1}\right)$, we have

$$
\lim _{n \rightarrow \infty} \int_{0}^{2 \pi} \frac{G\left(x_{n}\right)-e(t) x_{n}}{\left\|x_{n}\right\|^{2}} d t=0
$$

By $\left(\mathrm{H}_{2}\right)$, we get

$$
\lim _{n \rightarrow \infty} \sum_{j=1}^{p} \frac{J_{j}\left(t_{j}, x_{n}\left(t_{j}\right)\right)}{\left\|x_{n}\right\|^{2}}=0
$$

From (3.26) and the definition of $\varphi$, we obtain

$$
\limsup _{n \rightarrow \infty}\left[\frac{1}{2} \int_{0}^{2 \pi} \frac{x_{n}^{\prime 2}-m^{2} x_{n}^{2}}{\left\|x_{n}\right\|^{2}} d t-\int_{0}^{2 \pi} \frac{G\left(x_{n}\right)-e(t) x_{n}}{\left\|x_{n}\right\|^{2}} d t+\sum_{j=1}^{p} \frac{J_{j}\left(t_{j}, x_{n}\left(t_{j}\right)\right)}{\left\|x_{n}\right\|^{2}}\right] \leq 0
$$

For $x \in H^{+}$, we get

$$
\int_{0}^{2 \pi} x^{\prime 2} d t \geq m^{2} \int_{0}^{2 \pi} x^{2} d t
$$

Hence, for $x \in H^{+}$, we have

$$
\int_{0}^{2 \pi}\left(x^{\prime 2}-m^{2} x^{2}\right) d t \geq 0
$$

The equality in (3.30) holds only for

$$
x=\frac{1}{\sqrt{\left(m^{2}+1\right) \pi}} \sin (m t+\theta), \quad \theta \in \mathbb{R} .
$$


Set $y_{n}=\frac{x_{n}}{\left\|x_{n}\right\|}$. There exists $y_{0} \in H^{+}$such that $y_{n} \rightarrow y_{0}$ weakly in $H$. Due to the compact imbedding $H \hookrightarrow L^{2}(0,2 \pi)$, going to a subsequence, $y_{n} \rightarrow y_{0}$ in $L^{2}(0,2 \pi)$. Then (3.27), (3.28), (3.29) and (3.30) imply that

$$
y_{0}=\frac{1}{\sqrt{\left(m^{2}+1\right) \pi}} \sin (m t+\theta), \quad \theta \in \mathbb{R} .
$$

By (3.26), we have, for $n$ large enough,

$$
\frac{1}{2} \int_{0}^{2 \pi} \frac{x_{n}^{\prime 2}-m^{2} x_{n}^{2}}{\left\|x_{n}\right\|} d t-\int_{0}^{2 \pi} \frac{G\left(x_{n}\right)-e(t) x_{n}}{\left\|x_{n}\right\|} d t+\sum_{j=1}^{p} \frac{J_{j}\left(t_{j}, x_{n}\left(t_{j}\right)\right)}{\left\|x_{n}\right\|} \leq \frac{c_{+}}{\left\|x_{n}\right\|}
$$

It follows from $x_{n} \in H^{+}$that

$$
\int_{0}^{2 \pi} \frac{x_{n}^{\prime 2}-m^{2} x_{n}^{2}}{\left\|x_{n}\right\|} d t \geq 0
$$

From (3.31) and (3.32), we get, for $n$ large enough,

$$
\frac{c_{+}}{\left\|x_{n}\right\|} \geq-\int_{0}^{2 \pi} \frac{G\left(x_{n}\right)-e(t) x_{n}}{\left\|x_{n}\right\|} d t+\sum_{j=1}^{p} \frac{J_{j}\left(t_{j}, x_{n}\left(t_{j}\right)\right)}{\left\|x_{n}\right\|}
$$

Passing to the limits and using an argument similarly as in the proof of Lemma 3.1, we get

$$
2[G(+\infty)-G(-\infty)] \geq \int_{0}^{2 \pi} e(t) \sin (m t+\theta) d t+\sum_{j=1}^{p} K_{j}\left(t_{j}\right) \sin \left(m t_{j}+\theta\right),
$$

which is a contradiction to $\left(\mathrm{H}_{3}^{-}\right)$. This completes the proof.

\section{Competing interests}

The author declares that they have no competing interests.

\section{Author's contributions}

The author has contributed in obtaining new results and written the whole article.

\section{Acknowledgements}

The author would like to express their thanks to the editor of the journal and the referees for their careful reading of the first draft of the manuscript and making many helpful comments and suggestions which improved the presentation of the paper. This research was supported by the National Natural Science Foundation of China (11401274), Science and Technology Landing Project of colleges and universities in Jiangxi Province (KJLD14092) and Natural Science Foundation Project of Science and Technology Department of Jiangxi Province (20132BAB201012, 20142BDH80027).

Received: 16 September 2014 Accepted: 14 October 2014 Published online: 31 October 2014

\section{References}

1. Lazer, AC, Leach, DE: Bounded perturbations of forced harmonic oscillators at resonance. Ann. Mat. Pura Appl. 82(4), 49-68 (1969)

2. Capietto, A, Wang, Z: Periodic solutions of Liénard equations with asymmetric nonlinearities at resonance. J. Lond. Math. Soc. 68, 119-132 (2003)

3. Landesman, EM, Lazer, AC: Nonlinear perturbations of linear elliptic boundary value problems at resonance. J. Math. Mech. 19, 609-623 (1970)

4. Tomiczek, P: The Duffing equation with the potential Landesman-Lazer condition. Nonlinear Anal. 70(2), 735-740 (2009)

5. Wang, Z: Lazer-Leach type conditions on periodic solutions of Liénard equation with a deviating argument at resonance. Abstr. Appl. Anal. 2013, Article ID 906972 (2013) 
6. Bogun, I: Existence of weak solutions for impulsive $p$-Laplacian problem with superlinear impulses. Nonlinear Anal,, Real World Appl. 13(6), 2701-2707 (2012)

7. Bonanno, G, Bella, BD, Henderson, J: Existence of solutions to second-order boundary-value problems with small perturbations of impulses. Electron. J. Differ. Equ. 2013, 126 (2013)

8. Bonanno, G, Bella, BD, Henderson, J: Infinitely many solutions for a boundary value problem with impulsive effects. Bound. Value Probl. 2013, 278 (2013)

9. Ding, W, Qian, D: Periodic solutions for sublinear systems via variational approach. Nonlinear Anal., Real World Appl. 11(4), 2603-2609 (2010)

10. Lakshmikantham, V, Bainov, DD, Simeonov, PS: Theory of Impulsive Differential Equations. World Scientific, Singapore (1989)

11. Nieto, JJ, O'Regan, D: Variational approach to impulsive differential equations. Nonlinear Anal., Real World Appl. 10(2), 680-690 (2009)

12. Qian, D, Li, X: Periodic solutions for ordinary differential equations with impulsive effects. J. Math. Anal. Appl. 303, 288-303 (2005)

13. Sun, $H, L i, Y$, Nieto, JJ, Tang, Q: Existence of solutions for Sturm boundary value problem of impulsive differential equations. Abstr. Appl. Anal. 2012, Article ID 707163 (2012)

14. Sun, J, Chu, J, Chen, H: Periodic solution generated by impulses for differential equations. J. Math. Anal. Appl. 404 , 562-569 (2013)

15. Tian, Y, Ge, W: Applications of variational methods to boundary value problem for impulsive differential equations. Proc. Edinb. Math. Soc. 2, 509-527 (2008)

16. Xiao, J, Nieto, JJ, Luo, Z: Multiplicity of solutions for nonlinear second order impulsive differential equations with linear derivative dependence via variational methods. Commun. Nonlinear Sci. Numer. Simul. 1, 426-432 (2012)

17. Zhang, H, Li, Z: Variational approach to impulsive differential equations with periodic boundary conditions. Nonlinear Anal., Real World Appl. 11(1), 67-78 (2010)

18. Zhang, Z, Yuan, R: An application of variational methods to Dirichlet boundary value problem with impulses. Nonlinear Anal., Real World Appl. 11(1), 155-162 (2010)

19. Rabinowitz, PH: Minmax Methods in Critical Point Theory with Applications to Differential Equations. CBMS Reg. Conf. Ser. in Math., vol. 65. Am. Math. Soc., Providence (1986)

20. Mawhin, J, Willem, M: Critical Point Theory and Hamiltonian Systems. Springer, Berlin (1989)

21. Fučík, S: Solvability of Nonlinear Equations and Boundary Value Problems. Reidel, Dordrecht (1980)

doi:10.1186/s13661-014-0233-0

Cite this article as: Li: Lazer-Leach type condition for second order differential equations at resonance with impulsive effects via variational method. Boundary Value Problems 2014 2014:233.

\section{Submit your manuscript to a SpringerOpen ${ }^{\circ}$ journal and benefit from:}

- Convenient online submission

Rigorous peer review

- Immediate publication on acceptance

- Open access: articles freely available online

- High visibility within the field

- Retaining the copyright to your article 